This is the peer reviewed version of the following article:

Otte M.A., Solis-Tinoco V., Prieto P., Borrisé X., Lechuga L.M., González M.U., Sepulveda B.. Tailored Height Gradients in Vertical Nanowire Arrays via Mechanical and Electronic Modulation of Metal-Assisted Chemical Etching. Small, (2015). 11. : 4201 - . 10.1002/smll.201500175,

which has been published in final form at https://dx.doi.org/10.1002/smll.201500175. This article may be used for non-commercial purposes in accordance with Wiley Terms and Conditions for Use of Self-Archived Versions. 


\section{Tailored height gradients in vertical nanowire arrays via mechanical and electronic modulation of metal assisted chemical etching}

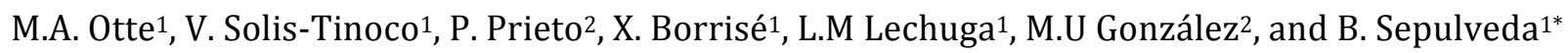

1 Institut Catala de Nanociencia i Nanotecnologia (ICN2), Consejo Superior de Investigaciones Científicas (CSIC) \& CIBER-BBN, Campus UAB, Bellaterra, 08193 Barcelona, Spain

2 IMM-Instituto de Microelectrónica de Madrid (CNM-CSIC), Isaac Newton 8, PTM, E-28760 Tres Cantos, Madrid, Spain

* Corresponding author: borja.sepulveda@cin2.es

\section{Keywords}

Nanofabrication, height gradients, high aspect-ratio vertical nanowires, metal assisted chemical etching, metamaterials 


\section{Abstract}

In current top-down nanofabrication methodologies the design freedom is generally constrained to the two lateral dimensions, and is only limited by the resolution of the employed nanolithographic technique. However, nanostructure height, which relies on certain mask-dependent material deposition or etching techniques, is usually uniform, and on-chip variation of this parameter is difficult and generally limited to very simple patterns. Herein, we present a novel nanofabrication methodology that enables the generation of high aspect-ratio nanostructure arrays with height gradients in arbitrary directions by a single and fast etching process. Based on metal-assisted chemical etching using a catalytic gold layer perforated with nanoholes, we demonstrate how nanostructure arrays with directional height gradients can be accurately tailored by: i) the control of the mass transport through the nanohole array, ii) the mechanical properties of the perforated metal layer, and iii) the conductive coupling to the surrounding gold film to accelerate the local electrochemical etching process. The proposed technique, enabling 20-fold on-chip variation of nanostructure height in a spatial range of a few microns, offers a new tool for the creation of novel types of nano-assemblies and metamaterials with interesting technological applications in fields such as nanophotonics, nanophononics, microfluidics or biomechanics. 
Top-down nanofabrication provides several routes to create nanostructured substrates whose material- and structure-specific properties have led to many new technological applications. These nanofabrication methods typically rely on the creation of a 2D mask, whose creative design is solely limited by the resolution of the employed lithographic technique, turning the cross-sectional area of the nanostructure into a precisely tunable on-chip variable. However, despite being accurately controllable, the nanostructure height is generally homogeneous across the chip, restricting its use as a versatile parameter. Height offers an additional variable to modify the optical, mechanical, magnetic and electronic properties of the nanostructures. For example, it can be employed to finetune the optical resonances in metal and dielectric nanostructures,[1-3] the spring constant and mechanical resonance frequency of nanopillars,[4] the thermal conductance in silicon nanowires,[5] or the magnetic behavior in ferromagnetic nanowires.[6] Therefore, the ability to vary in a controlled way the height within an array of nanostructures could offer a novel degree of freedom to engineer metamaterials and nanodevices with unprecedented optical, acoustic, thermal, electronic, mechanical or magnetic properties. These innovative applications would require the creation of nanostructure arrays with height gradients at the nano/micrometer-scale in predefined directions. However, current top-down nanofabrication techniques offer very limited capabilities to vary the height of the nanostructures. Some exceptions exist, such as mechanically shuttered physical vapor deposition, 3D gray-scale lithography[7] or 3D two-photon polymerization,[8, 9] but these techniques are generally restricted to simple linear height gradients and low aspect ratios, or exhibit poor lateral resolutions. To surpass these design limitations, herein we present a novel method to fabricate arrays of high aspect-ratio vertical silicon nanowires with precisely adjustable height gradients in arbitrary directions,[10] which enables the creation of nanostructures with a 20 -fold on-chip height variation (from $c a .50$ to $c a .1000 \mathrm{~nm}$ ) within a spatial range of a few microns via a single and fast etching process. 
This new approach relies on a process denoted as metal assisted chemical etching (MACE).[11] In general, MACE exploits the catalytic activity of a noble metal that physically contacts both silicon and an etching solution composed of $\mathrm{HF}$ and an oxidizing agent, typically $\mathrm{H}_{2} \mathrm{O}_{2}$. During MACE, the $\mathrm{H}_{2} \mathrm{O}_{2}$ is first reduced at the cathode (i.e. the liquid/metal interface) and, at the anode (i.e. the metal/silicon interface), the generated electronic holes $\left(h^{+}\right)$are injected into the silicon, causing its oxidation and subsequent dissolution by $\mathrm{HF}$ via the formation of soluble $\mathrm{H}_{2} \mathrm{SiF}_{6}$. Therefore, when a thin metal film perforated with nanoholes is used as catalyst, fast silicon etching is assured underneath the metal, while the exposed silicon inside the holes is etched at a much lower rate, hence assuring the formation of vertical 3D nanostructures that can reach very high aspect ratios.[11] Searching for ways to control and tune the height of these silicon nanostructures, herein we demonstrate that the MACE rate is accurately regulated by the balance of mechanical and van der Waals forces acting in the perforated metal layer, the mass transport rate through the nanohole array, and its conductive coupling to the surrounding metal. This regulation of the MACE process, enables the local nano-scale control of the etching rate to achieve arrays of vertical nanowire with height gradients in any direction in a single, fast and cost effective process.

The main findings of this work are gathered in Fig. 1, which illustrates three different catalytic conditions to spatially modulate the MACE rate: i) a circular nanohole array in a $20 \mathrm{~nm}$ thick gold layer that is mechanically and electrically connected to the surrounding gold film, resulting in a nanopillar array with a radial height gradient; ii) a nanohole array that is mechanically and electrically isolated from the surrounding metal, generating a nanopillar array with a homogenous height, but showing a four-fold reduction of the MACE rate, and iii) a nanohole array that is only mechanically and electrically connected to the surrounding metal via a gold nanochannel, yielding a nanopillar array with approximately uniform heights and an etching rate similar to that at the center of the fully connected array. 


\section{Mechanical, geometrical and electronic control of MACE rate to generate height gradients}

The mechanisms behind this peculiar etching behavior can be explained as follows. First, the injection of $h^{+}$through the metal oxidizes the first silicon layer underneath the metal. This enables HF, which can penetrate underneath the gold layer through the nanoholes, to dissolve the oxidized silicon. Once underneath the metal, HF can solely penetrate a certain distance in the outward radial direction of the nanoholes (ca. $700 \mathrm{~nm}$ ), up to a point where the reaction is stopped due to a lack of mass transport. During MACE, the nanoholes at the perimeter of the array in Fig. 1a become a stopping barrier for the reaction, while the surrounding homogeneous gold film forms a mechanical clamp for the perforated metal film, which can mechanically behave as an elastic or plastic membrane. Once the first silicon layers are etched, there are two main forces that drive the mechanics of the etching process (Fig. 2a): the attractive van der Waals force between the gold film and the silicon substrate, $[12,13]$ and the mechanical restoring force of the perforated membrane. The former is given by:

(eq. 1 )

$$
F_{v d W}=\frac{C}{6 \pi s^{3}} A(1-\phi)
$$

where $C$ is the Hamaker constant, $s$ the separation between the gold layer and the substrate, $A$ the total membrane area, and $\phi$ the fraction of membrane covered by the nanoholes. Such fraction can be approximated by $\phi \approx \frac{\pi}{4} \frac{\mathrm{D}_{\mathrm{nh}}^{2}}{\mathrm{P}^{2}}$ (Supporting Information S1), where $D_{n h}$ is the nanohole diameter and $P$ the periodicity.

The mechanical restoring force in the elastic regime of a clamped, circular and perforated membrane, subjected to a homogeneous load can be approximated by:[14]

$$
\text { (eq. } 2) \quad F_{\text {mech }}=-\frac{64 \pi \cdot \mathrm{E} \cdot \mathrm{t}^{3}}{3 \cdot \mathrm{D}^{2}\left(1-v^{2}\right)}(1-\phi) H_{\text {center }}
$$


where $E$ and $v$ are the Young's Modulus and Poisson ratio of the metal, respectively, $t$ the metallic layer thickness, $D$ the diameter of the array, and $H_{\text {center }}$ the deformation depth at the center of the array (Fig. 2b), which can be easily retrieved by measuring the height of the nanostructures.

By comparing equations 1 and 2, one can first observe that both forces have the same dependence on the fraction of membrane covered by nanoholes. As a result, the nanohole diameter and periodicity should not influence the mechanics of the etching process, even though we will show below that the edge-to-edge distance between nanoholes plays a key role to control the mass transport, and thereby the local etching rate.

We first theoretically analyze the forces governing MACE mechanics keeping a constant $\phi\left(D_{n h}=\right.$ $200 \mathrm{~nm}, P=400 \mathrm{~nm}$ ). We consider two possible scenarios for $F_{v d w:}$ the interaction between gold and silicon or between gold and silicon oxide, which are represented by two different Hamaker constants, $C_{A u S i}=2.710^{-19} \mathrm{~J}$ and $C_{A u S i O 2}=2.510^{-20} \mathrm{~J}$, respectively,[13] that take into account the presence of the liquid (i.e. water) between both surfaces. The gold-silicon interaction represents the upper limit of $F_{v d W}$, since either the formation of a nanoporous silicon structure or an intermediate $\mathrm{SiO}_{2}$ layer below the metal during the etching process will cause a substantial decrease in the force amplitude. The formation of intermediate interfacial $\mathrm{SiO}_{2}$ can be assumed at high $h^{+}$injection currents,[11,12] which is the case when the membrane is electrically connected to the surrounding metal film (Figs. 1a and 1c), as we will point out below.

The continuation of MACE requires that $F_{v d W}>F_{\text {mech }}$ to maintain the catalyst layer and the silicon substrate in mutual contact. Figure 2 compares the magnitude of $F_{v d W}$ and $F_{m e c h}$ as a function of the separation distance $(s)$ between the gold layer and the silicon substrate, and of the mechanical deformation at the center of the membrane $\left(H_{\text {center }}\right)$, respectively, for different diameters $(D)$ and thicknesses $(t)$ of the metal membrane. We can assume that $F_{v d W}$ is constant for a given $\mathrm{HF}$ and $\mathrm{H}_{2} \mathrm{O}_{2}$ concentration, i.e. the factor $s$ is kept approximately constant during the etching process. In 
contrast, $F_{\text {mech }}$ linearly increases with the etching depth. The calculations of Fig. 2 show two different regimes. When the gold layer thickness is $20 \mathrm{~nm}$ and the diameter of the membrane is larger than $10 \mu \mathrm{m}, F_{v d W} \gg F_{\text {mech }}$ considering both $\mathrm{Au} / \mathrm{Si}$ and $\mathrm{Au} / \mathrm{SiO}_{2}$ van der Waals interactions. In these conditions $F_{\text {mech }}$ can be practically neglected and the mechanics of the etching process will be driven by van der Waals interactions. As a result of such large force difference, we expect that the etching process will be able to mechanically deform the gold membrane and to overcome its ultimate strength, thus leading to the uncontrolled rupture of the membrane. In contrast, for $D$ smaller than $10 \mu \mathrm{m}$, or for gold layers thicker than $50 \mathrm{~nm}, F_{v d W}$ and $F_{\text {mech }}$ can be in the same range, and it is even possible to find values of $s$ and $H_{c e n t e r}$ for which $F_{v d W}=F_{m e c h}$. When this condition is fulfilled, the etching process will be stopped, since the gold layer will not be able to get closer to the silicon substrate to continue the catalytic oxidation. For a $100 \mathrm{~nm}$ thick gold layer, the calculations predict that $F_{\text {mech }}$ can be initially larger than $F_{v d W}$, which will prevent continuation of MACE after the first silicon layers are etched.

In the conditions of Fig. 1a, $F_{v d W}$ is substantially larger than $F_{m e c h}$, leading to the initial downward movement of the metal film at the center of the membrane. Due to the mechanical clamping, tensile stress is built up at the edge of the membrane, thereby mechanically deforming the catalytic metal sheet. As MACE continues, this effect slowly expands radially inwards. The result of this mechanically modulated etching process is an array of silicon nanopillars whose height increases towards the center of the membrane, generating a nanopillar array with a radial height gradient. Thus, as long as the metal layer is able to elastically or plastically deform, MACE will progress, adapting its shape to the underlying silicon substrate. Indeed, Figs. 1a and 1c, displaying a similar etching depth when both membranes are electrically connected to the surrounding metal film also confirm the negligible effect of $F_{\text {mech }}$ in these conditions. 
To gain a deeper control of the mechanics that govern the formation of these nanopillar arrays with height gradients, we have carried out a systematic analysis of the fundamental parameters $\left(t, D, D_{n h}\right.$, and $P$ ) that strongly influence the rigidity of the perforated membrane. Throughout this analysis we maintained the same MACE conditions $\left(10 \% \mathrm{HF}, 0.5 \% \mathrm{H}_{2} \mathrm{O}_{2}\right.$, 2-min etching time). To demonstrate the large influence of membrane rigidity on the etching rate, we first analyze nanohole arrays with different metal thickness and similar values of $D, D_{n h}$ and $P$. As Fig. 3a indicates, very rigid metal membranes $(t=100 \mathrm{~nm})$ exhibit negligible etching, whereas there is a drastic increase of the etching rate at the center of the membranes as $t$ is reduced to $20 \mathrm{~nm}$. These results confirm that $F_{m e c h}$ is initially larger than $F_{v d W}$ in $100 \mathrm{~nm}$ thick membranes, thereby preventing the continuation of MACE. The height gradients in the nanostructured arrays are maximized for the thinnest metal layers, motivating the selection of $t=20 \mathrm{~nm}$ for the analysis of the remaining membrane parameters.

The second parameter that strongly influences $F_{\text {mech }}$ is the membrane diameter (Fig 3b). For every combination of $D_{n h}$ and $P$, the etching rate increases as $D$ becomes larger, leading to nanopillars with larger height at the array center. Interestingly, for $D>20 \mu \mathrm{m}$ the observed growth exhibits signs of saturation, whereas for smaller array diameters $(D \leq 20 \mu \mathrm{m})$ a steep monotonic decrease of $H_{c e n t e r}$ is measured as $D$ is reduced. Such steep decrease in the MACE rate is a clear signature of the increasing influence of $F_{m e c h}$, which is consistent with the predictions of Fig. 2, showing that $F_{m e c h}$ and $F_{v d W}$ can be of the same order of magnitude for $D=5 \mu \mathrm{m}$ and $t=20 \mathrm{~nm}$.

From the morphological characterization of the nanopillar arrays it is possible to estimate the total mechanical deformation of the metal membrane during MACE. Such deformation is obtained by calculating the incremental change in area of the perforated membrane $(d A)$ that, assuming that the bent metal film can be approximated by a spherical capped geometry, can be expressed as (Supporting information S2): 


$$
\mathrm{dA}=\frac{4 \mathrm{H}_{\text {center }}^{2}}{\left(1-\frac{\pi_{\mathrm{nh}}^{2}}{4 \mathrm{P}^{2}}\right) \mathrm{D}^{2}}
$$

Figure $3 \mathrm{~b}$ shows that larger arrays $(D>20 \mu \mathrm{m})$ experience low deformations $(d A<0.5 \%)$ after a 2min-etch. However, for $D<20 \mu \mathrm{m}, d A$ increases exponentially as $D$ becomes smaller. In general, the mechanical deformation is maximized for the smallest nanopillar arrays ( $D=5 \mu \mathrm{m})$, proclaiming that these arrays are subjected to the largest amount of mechanical tension. In fact, these arrays are the first ones that fracture for slightly increased etching times (larger than $2 \mathrm{~min}$, Supporting information S3). As can be observed in these figures, $d A$ is limited to $3 \%$, thus defining a rough limit for the maximum plastic deformation of these arrays for $20 \mathrm{~nm}$ thick gold layers. For longer etching times all metal membranes ultimately undergo mechanical rupture, confirming that $F_{v d W}>F_{m e c h}$ in all the analyzed cases when the film thickness is $20 \mathrm{~nm}$.

Interestingly, high resolution SEM imaging enables determining the separation distance between the membrane and the silicon substrate during MACE, by measuring the distance between the end of the metal film and the lateral surface of the nanopillar at its base (Fig. 2a). SEM images show that $s$ varies in a range from 7 to $10 \mathrm{~nm}$ (Supporting information S4). These values are substantially larger than those deduced by Lai et al.,[13] thus reflecting the highly amplified $h^{+}$injection current in our experimental conditions.

Thicker metal membranes can be used to generate mechanical conditions in which $F_{\text {mech }}$ matches $F_{v d W}$ during the etching process, thereby stopping MACE at a specific etching depth (Supporting information S5). The possibility to reach the force equilibrium in $50 \mathrm{~nm}$ thick membranes has enabled us to extract important parameters, such as the van der Waals pressure $\left(\sigma_{v d w}\right)$ and the Hamaker constant for our etching conditions. These results indicate that $\sigma_{v d W}$ is in the range of 2 $\mathrm{kPa}$, i.e. considerably lower than the estimations by Lai et al.,[13] which is consistent with the large $s$ values observed in the SEM images. Indeed, assuming an average $s=9 \mathrm{~nm}$, the retrieved Hamaker 
constant is $C=2.710^{-20} \mathrm{~J}$, which is very close to that expected for $\mathrm{Au} / \mathrm{SiO}_{2}$ interaction (Supporting information S5). These values confirm that: i) the $h^{+}$injection current is highly amplified, and ii) the etching process follows the intermediate formation of $\mathrm{SiO}_{2}$ layer.

The nanohole diameter and periodicity also play an important role in the MACE rate modulation (Fig. 3b). Increasing $D_{n h}$ or reducing $P$ results in higher etching rates. We have previously argued that the metal filling fraction should not affect the mechanics of MACE. However, both $D_{n h}$ and $P$ have a strong impact on the mass transport dynamics and thereby on the etching rate. Figure 1a shows that MACE is stopped at the perimeter of the membrane, and the maximum penetration distance of the etching solution is $c a .700 \mathrm{~nm}$. Therefore, the metal distance between nanoholes is the key parameter to control the mass transport rate, and this parameter can be represented by the edge-to edge distance $\left(d_{e e}\right)$ between nanoholes. Figure 3c depicts the etching rate as a function of $d_{e e}$ in two cases in which the mechanical force have a negligible $(D=40 \mu \mathrm{m})$ or substantial influence $(D$ $=5 \mu \mathrm{m}$ ) on MACE mechanics. Both cases show the $d_{e e^{-1}}$ dependence expected from Fick's law of diffusion (Supporting information S6). Consequently, $d_{e e}$ reduction permits a faster mass interchange underneath the metal membrane and a local increase of the MACE rate, thereby providing another parameter to engineer the height gradients in the nanostructure arrays. For $D=$ $5 \mu \mathrm{m}$, the mechanical restoring force induces a decrease in the slope of the $d_{e e^{-1}}$ dependence. Such change in the slope is not due to a variation in HF diffusivity, but to the drop in the concentration gradient underneath the metal as a result of the slower conversion rate of $\mathrm{HF}$ and $\mathrm{Si}$ into $\mathrm{H}_{2} \mathrm{SiF}_{6}$. From Fig. 3c we can determine the diffusion coefficients of HF across the membrane, which are $12 \cdot 10^{3} \mathrm{~nm}^{2} / \mathrm{s}$ and $4 \cdot 10^{3} \mathrm{~nm}^{2} / \mathrm{s}$ for $D$ equal to $40 \mu \mathrm{m}$ and $5 \mu \mathrm{m}$, respectively. Such reduction in the diffusion coefficient clearly reflects the influence that the mechanical restoring force exerts on the etching rate. 
Next to the mechanical and mass transport control, we discovered another interesting parameter to fine-tune the MACE rate and to tailor the height gradients. Mechanical isolation of nanohole arrays assures uniform nanopillar heights (see Fig. 1b) due to the suppression of the clamping provided by connection to the rest of the metal film. However, surprisingly, despite the absence of the mechanical restoring force, nanopillar heights are four-fold lower than those of mechanically constrained membranes. Even more strikingly, the presence of just a tiny metal contact between the array and the surrounding metal fully re-establishes the MACE rate across the whole array (Fig. 1c). This observation is explained by closely analyzing the charge transfer phenomena that occur during MACE. When MACE starts, $h^{+}$are injected across the entire silicon surface, thereby creating a thin layer of oxidized silicon below the metal. Nevertheless, it is only at the perforated membrane, where the HF can penetrate through the nanoapertures, and dissolve the oxidized silicon. At this point, direct contact of the metal layer with non-oxidized silicon only exists underneath the perforated membrane, spatially restricting further injection of $h^{+}$-and the MACE process- to this very region. Interestingly, electric contact between the nanohole array and the surrounding metal enables $h^{+}$generated across the entire gold surface to move through the conduction band of the metal, and to be injected into the silicon underneath the perforated membrane. The latter assures an amplified $h^{+}$density at the etching front, and, since the MACE rate strongly depends on this density, $[15,16]$ an enhanced MACE rate is expected to keep the charge balance in the electrochemical process. As a result, all metal that contacts the etching solution acts as a cathode, but only the small metal membrane is continuously acting as anode. Under these conditions the surrounding metal behaves as a pool of $h^{+}$to amplify the MACE rate by a factor of four. In contrast, the closed metal-free frame around the nanohole arrays prevents electronic holes from reaching the perforated metal membrane. In this case, the cathode area is shrunk down, coinciding in size with the metal membrane, and thus, with the anode. Consequently, only the $h^{+}$injected at the membrane can contribute to MACE, yielding a much lower hole density at the etching front, and 
hence, a lower etching rate. Nevertheless, when the metal-free frame contains a narrow metal channel (Fig. 1c) the electrical contact is re-established, thereby enabling the full contribution of the $h^{+}$generated at the surrounding metal to amplify the MACE process again.

These results unambiguously confirm that the MACE rate is heavily conditioned by whether or not the nanohole array is isolated from the surrounding gold film and, what is even more interesting from a technological perspective, that the final height gradients can be accurately controlled by the total metal area that contacts the perforated membrane. To demonstrate such capability, we fabricated perforated metal membranes surrounded by frames of increasing diameter $\left(D_{\text {frame }}\right)$, hence enabling relative variations of the cathode area with respect to a constant sized anode $(D=$ $10 \mu \mathrm{m}$, Fig. 4a). Figure $4 \mathrm{~b}$ displays $H_{\text {center }}$ as a function of $D_{\text {frame, }}$ confirming that the etching rate, and thus the height gradients, grows as $D_{\text {frame }}$ increases. This growth is extremely steep for $D_{\text {frame }} \leq 50$ $\mu \mathrm{m}$. Further increase of $D_{\text {frame }}$ results in saturation of the measured nanopillar heights, slowly approaching the etching rate of the arrays connected to the surrounding metal film. When $D_{\text {frame }}=$ $50 \mu \mathrm{m}$, the ratio between the cathode and the anode areas is a factor 24 , whereas the amplification of the etching rate is approximately four-fold. Therefore, $h^{+}$concentration can be amplified 4-fold at the etching front. These results indicate that the increase of $h^{+}$concentration at the etching front does not follow a linear relationship with respect to the surrounding metal area. Instead, these results suggest the existence of an effective capture distance of approximately $40 \mu \mathrm{m}$ around the metal membrane that the injected $h^{+}$can travel to amplify the MACE process. The high $h^{+}$ concentration amplification also provides an additional indication that the electrochemical reaction can follow the formation of an intermediate $\mathrm{SiO}_{2}$ layer, thereby substantially decreasing the Van der Waals force. 


\section{Fabrication of gradients in arbitrary directions, and gradient amplification by controlled metal rupture}

So far, we have demonstrated the generation and control of nanostructures arrays with radial height gradients, however, through geometrical manipulation of the nanohole array, other exciting nanopillar assemblies can be made. An eye-catching example is provided by a ring-shaped array (Fig. 5a), in which 2 circular clamping regions run along its inner and outer radius. Consequently, the resulting nanopillar arrangement describes a more complex gradient displaying the highest nanopillars along the circumference that is approximately equidistant to the inner and outer radius.

Another attractive method to tailor the height gradient direction is the controlled release of the mechanical tension at specific regions of the nanohole array perimeter using metal-free frames. Through careful geometric design of the nanohole arrays and frames, height gradients in arbitrary directions can then be created, as illustrated in Fig. 5. Herein, two squared arrays of nanopillars are shown, Fig. $5 b$ and $5 c$, in which the nanohole array is flanked by a continuous frame along 3 or 2 opposite sides, respectively. The metal membrane that is only clamped at one side, results in a nanopillar array that exhibits a 1D height gradient with gradually increasing pillar heights that go from the clamp to the opposite frame. Interestingly the generated nanopillars are slightly inclined, as during MACE the metal film describes a hinge-like trajectory. Such inclination and the height gradient can be drastically increased in this configuration by exploiting the free plastic deformation of the metal membrane when it is clamped at just one border. In contrast, in the configuration of Fig. 5c, MACE results in a nanopillar array with a linear gradient in two opposite directions that exhibit the highest pillars along the line that is equidistant to the metal clamps. In this case, the mechanical clamps restrict the maximum achievable height gradient to the largest plastic deformation of the metal film before the rupture (ca. 3\% for $20 \mathrm{~nm}$ thick gold layers). 
Consequently, by combining the shape of the metal membrane with metal-free frames to release the mechanical stress, it is possible to generate height gradients in any desired direction.

Finally, to significantly increase the height gradients limited by the plastic deformation of the metal film, we show how the nanohole arrays can be designed to intentionally induce a controlled rupture of the metal layer at specific points. This effect can be achieved by the generation of regions with faster mass transport (i.e. higher etching rate) within the metal membrane, by locally increasing the size of the nanoholes or reducing their periodicity. As illustrated in Fig. 5d, the higher degree of perforation along the central line of nanoholes results in a controlled rupture of the metal membrane along this line during MACE, breaking it in two equal parts. After the rupture, both array halves are solely clamped from their respective lateral sides, causing them to free bend as if they were two hinges. As a result, the obtained nanopillars are vertical in the top part, and there is a sudden and strong change of the inclination of the nanopillars at a specific height, which corresponds to the position of the metal membrane where it broke in two parts. But more importantly, the suppression of the two-sided clamping assures a much faster etching rate, yielding much larger nanopillars.

This innovative nanofabrication strategy to generate high aspect ratio nanostructures with a 20fold on-chip height variation in a single and very fast MACE process offers significant improvements in terms of spatial resolution, size, shape (Supporting information S7) and height range compared to the very few existing nanofabrication techniques that enable the generation of height gradients. This nanofabrication method can find remarkable technological applications. From the nanophotonics perspective, the silicon nanopillar arrays with height gradients could be employed to develop pioneering metalenses.[17-20] The arrays with radial height gradients could be used to focus collimated light into small spots, and as lenses whose focal length and spectral response can be tuned on-chip through the height gradient, diameter and periodicity of the nanopillars. Since the 
MACE process is not restricted to circular nanoholes, the change of the nanohole shape can yield highly anisotropic nanopillars (Supporting information S7) with potential polarization dependent optical effects. Moreover, the geometry of the nanopillar array with height gradients could be used to focus the light in arbitrary shapes (such as lines, rings...). On the other hand, the variation of the mechanical properties of the nanopillars within the arrays, i.e. the spring constant and resonant frequency, can also find attractive nanophononic applications.[21-23] In the particular case of Fig. 3B $\left(P=300 \mathrm{~nm}, D_{n h}=220 \mathrm{~nm}, D=40 \mu \mathrm{m}\right)$, the resonant frequency of the nanopillars is fine-tuned from $0.3 \mathrm{GHz}$ to $5 \mathrm{GHz}$ in a distance as short as $20 \mu \mathrm{m}$. This large variation could be employed to control the propagation direction and even to focus surface and volumetric acoustic phonons. To add more technological interest, the silicon nanopillar arrays with height gradients can be replicated in other materials, such as polymers (Supporting information S8), metals..., which greatly expands the range of optical, mechanical, electronic and magnetic properties that can be achieved for the development of novel nanodevices and applications. 


\section{Methods: Nanofabrication of the perforated metal membranes and MACE parameters}

First, a titanium/gold bilayer $(0.5 \mathrm{~nm} / 20 \mathrm{~nm})$ is evaporated on top of piranha-cleaned silicon wafers (100) via electron beam physical vapor deposition (EBPVD), after which a polymethyl methacrylate (PMMA $950 \mathrm{~K}, 6 \%$ in Anisole) resist layer is spin coated on top of it (4000 rpm: thickness $\approx 300 \mathrm{~nm}$ ). Then, electron beam lithography (EBL) is used to pattern circular, rectangular or ring-shaped arrays of nanoholes into the resist, using the dose that defines a single nanohole as the variable that controls the hole diameters. The frames (width $\approx 500 \mathrm{~nm}$ ) that surround the nanohole arrays are included during the EBL patterning. After EBL exposure the resist is developed during $40 \mathrm{~s}$ in a mixture of methyl isobutyl ketone (MIBK) and isopropyl alcohol (IPA) at a ratio of $1: 3$, respectively, a process that is stopped by subsequently immersing the chip in IPA, after which it is blown dry under a gentle flow of $\mathrm{N}_{2}$. Next, the patterned PMMA layer is used as a mask for the removal of the exposed gold inside the holes - and if present, also the frames - by means of a directional argon sputtering treatment (ICP RIE, power $1000 \mathrm{~W}$, chuck power $25 \mathrm{~W}$, pressure $1 \mathrm{~Pa}$, flow $20 \mathrm{sccm}$, time $50 \mathrm{~s}$ ). Although during this process the approximately $300 \mathrm{~nm}$ thick exposed PMMA layer is strongly damaged, it is still sufficiently thick in order to act as a buffer that protects the gold that resides underneath it. Subsequently, the remaining PMMA is removed via an oxygen plasma (ICP RIE power $500 \mathrm{~W}$, pressure $1 \mathrm{~Pa}$, flow $20 \mathrm{sccm}$, time $240 \mathrm{~s}$ ), leaving a $20 \mathrm{~nm}$ thick hole-patterned layer of gold on top of the silicon substrate. Finally, silicon nanopillars are created via a highly anisotropic metal-assisted chemical etching protocol (MACE), through immersion of the sample into a mixture of $\mathrm{H}_{2} \mathrm{O}(89,5 \%), \mathrm{H}_{2} \mathrm{O}_{2}(0.5 \%)$ and $\mathrm{HF}(10 \%)$ for $2 \mathrm{~min}$. The MACE is stopped by immersing the samples in water. Finally, the samples are transferred to IPA to minimize capillary effects upon drying under a gentle flow of $\mathrm{N}_{2}$.

The use of this methodology for much thicker metal layers requires larger Ar sputtering times, and thus, PMMA buffer layers with increased thicknesses that protect the underlying gold during a longer time span. This condition strongly deteriorates our best possible EBL patterning resolution, 
making this technique unemployable for the nanohole diameter used throughout this work. Hence, for experiments that require gold layers thicker than $20 \mathrm{~nm}$, the nanohole arrays were patterned directly onto the metal film via Focused Ion Beam (FIB) patterning: titanium/gold bilayers were subjected to direct milling of an ion beam in order to create the nanohole arrays. We used a $30 \mathrm{kV}$ $\mathrm{Ga}^{+}$ion beam with a measured current of 6 pA. Nanostructures with lateral size smaller than 200 $\mathrm{nm}$ were milled in a single pass, while multiple (50) passes were performed for bigger structures to avoid metal redeposition. The dose needed to etch the full thickness of the metal layer was obtained from the following calibration of the gold etching rate $\left(r_{e}\right)$ : for single pass processes, $r_{e}=19.5$ $\mathrm{nm} \cdot \mathrm{cm}^{2} / \mathrm{mC}$, and for 50 passes ones, $r_{e}=18 \mathrm{~nm} \cdot \mathrm{cm}^{2} / \mathrm{mC}$. The beam was scanned in a meander way for rectangular-shaped nanoholes, and in a concentric path for circular ones. To mimic the conditions of the EBL-based samples, all chips were coated after FIB exposure with a layer of PMMA and subjected to an identical oxygen plasma treatment. Finally, the samples underwent the same MACE process as described above. 


\section{Acknowledgements}

Authors acknowledge the financial support of Spanish MINECO projects MAT2011-12645-E, TEC2012-34280, MAT2011-29194-C02-01, and MAT2013-48628-R. V. Solis-Tinoco acknowledges financial support from The National Council for Science and Technology (CONACyT), Mexico. ICN2 acknowledges support of the Spanish MINECO through the Severo Ochoa Centers of Excellence Program under Grant SEV-2013-0295.

\section{Author contribution}

BS conceived the idea of the nanostructures arrays with height gradients in arbitrary directions and the suitable nanofabrication process.

MAO, MUG and BS planned the experiments.

MAO, VST, PP, XB, MUG and BS carried out the nanofabrication processes.

MAO and BS made the SEM characterization and structural analysis.

MAO and BS wrote the manuscript.

All authors contributed to the revision of the manuscript.

\section{Competing Financial Interests}

Authors declare no competing financial interests. 


\section{Figure Captions}

Fig 1. Circular arrays of silicon nanopillars with or without a radial height gradient. (A, B, C) SEM images obtained at $\theta=30^{\circ}$ (top) and $\theta=75^{\circ}$ (bottom) of identical circular silicon nanopillar arrays $\left(D=10 \mu \mathrm{m}, D_{n h} \approx 150 \mathrm{~nm}, P=400 \mathrm{~nm}, t=20 \mathrm{~nm}\right)$, with (A) a radial height gradient $\left(H_{\text {center }} \approx\right.$ $330 \mathrm{~nm}$ ), or without a radial height gradient, obtained with either (B) a closed metal-free frame $\left(H_{\text {center }} \approx 80 \mathrm{~nm}\right)$, or $(\mathbf{C})$ a quasi-closed metal-frame with a metal channel width of approximately $500 \mathrm{~nm}\left(H_{\text {center }} \approx 340 \mathrm{~nm}\right)$. All panes contain schematics depicting the initial pattern of perforations in the catalytic metal layer.

Fig 2 Mechanics of the etching process. (A) Schematics displaying the forces governing the mechanics of the MACE process. The zoomed-in region shows the separation distance $s$ between the gold layer and the silicon substrate during MACE. (B) Comparison of theoretical van der Waals and mechanical forces. The continuous black, red, blue and magenta lines represent $F_{v d W}$ for $D$ equal to $5 \mu \mathrm{m}, 10 \mu \mathrm{m}, 20 \mu \mathrm{m}$ and $40 \mu \mathrm{m}$, respectively, assuming $\mathrm{Au} / \mathrm{Si}$ interaction. The dashed lines represent the same $F_{v d W}$ assuming $\mathrm{Au} / \mathrm{SiO}_{2}$ interaction. In $F_{\text {mech }}$ plot, the black, red, blue and magenta filled squared are the forces for membrane diameters $D$ equal to $5 \mu \mathrm{m}, 10 \mu \mathrm{m}, 20 \mu \mathrm{m}$ and $40 \mu \mathrm{m}$, respectively, when the metal thickness is $20 \mathrm{~nm}$. The open blue circles and triangles represent membranes with $20 \mu \mathrm{m}$ diameter and whose metal thickness are $50 \mathrm{~nm}$ and $100 \mathrm{~nm}$, respectively.

Fig 3. Bending mechanics of circular nanohole arrays, analyzed as a function of their geometrical properties. (A) Measured nanopillar height at arrays center $\left(H_{\text {center }}\right)$ for metal membranes as a function of the metal film thickness when the array diameter $(D)$ is $20 \mu \mathrm{m}$, the nanohole diameter $\left(D_{n h}\right)$ is $200 \mathrm{~nm}$ and the periodicity $(P)$ is $400 \mathrm{~nm}$. (B) Measured $H_{c e n t e r}$, when the 
gold film thickness is $20 \mathrm{~nm}$, examined as a function of $D$ and $D_{n h}$, for $P$ equal to $300 \mathrm{~nm}, 400 \mathrm{~nm}$ and $500 \mathrm{~nm}$. Calculated relative area deformation $(d A)$ during MACE in previous geometrical conditions. (C) Experimental etching rate at the array center as a function of the edge-to-edge distance $\left(d_{e e}\right)$ for metal membranes of $D$ equal to 5 and $40 \mu \mathrm{m}$ with $t=20 \mathrm{~nm}$, and their corresponding fit to Fick's Law of diffusion.

Fig. 4 Tunability of the MACE rate as a function of the available charge carriers during the

etching process. (A) SEM image that illustrates how nanopillar arrays can be isolated from the surrounding $20 \mathrm{~nm}$ thick gold film via the creation of a closed frame with a tunable diameter size, which in this particular case is given by $D_{\text {frame }}=50 \mu \mathrm{m}$. (B) Measured nanopillar height at array centers $\left(H_{\text {center }}\right)$ for arrays $(D=10 \mu \mathrm{m}, P=400 \mathrm{~nm}, \mathrm{t}=20 \mathrm{~nm})$ surrounded by closed frames, analyzed as a function of varying nanohole diameters $\left(D_{n h} \approx 150 \mathrm{~nm}\right.$ and $\left.D_{n h} \approx 200 \mathrm{~nm}\right)$ and frame diameters $\left(D_{\text {frame}}\right)$. The continuous lines, specified for each analyzed value of $D_{n h}$, correspond to the values of $H_{\text {center }}$ for identical nanohole arrays not surrounded by any frame.

Fig. 5 Nanopillar arrays with height gradients in arbitrary directions. (A) Nanopillar array with ring-shape gradient, obtained by MACE using a ring shape array of circular nanoholes. (B) Nanopillar array with 1D gradient achieved by the mechanical stress release at three sides of the square array of nanoholes. (C) Nanopillar with two opposite 1D gradients, exploiting the release of the mechanical stress at two opposite sides of a rectangular array of nanoholes. (D) Controlled rupture of the catalytic metal film by using a line of nanoholes with smaller periodicity, to amplify the nanopillar height gradient. 


\section{References}

1. Kabashin, A. V.; Evans, P.; Pastkovsky, S.; Hendren, W.; Wurtz, G. A.; Atkinson, R.; Pollard, R.; Podolskiy, V. A.; Zayats, A. V., Nat. Mater. 2009, 8 (11), 867-871.

2. Seo, K.; Wober, M.; Steinvurzel, P.; Schonbrun, E.; Dan, Y.; Ellenbogen, T.; Crozier, K. B., Nano Lett. 2011, 11 (4), 1851-1856.

3. Simovski, C. R.; Belov, P. A.; Atrashchenko, A. V.; Kivshar, Y. S., Adv. Mater. 2012, 24 (31), 4229-4248.

4. Gil-Santos, E.; Ramos, D.; Martinez, J.; Fernandez-Regulez, M.; Garcia, R.; San Paulo, A.; Calleja, M.; Tamayo, J., Nat. Nanotechnol. 2010, 5 (9), 641-645.

5. Hochbaum, A. I.; Chen, R.; Delgado, R. D.; Liang, W.; Garnett, E. C.; Najarian, M.; Majumdar, A.; Yang, P., Nature 2008, 451 (7175), 163-167.

6. Liu, Z.; Chang, P.-C.; Chang, C.-C.; Galaktionov, E.; Bergmann, G.; Lu, J. G., Adv. Funct. Mater. 2008, 18 (10), 1573-1578.

7. Piaszenski, G.; Barth, U.; Rudzinski, A.; Rampe, A.; Fuchs, A.; Bender, M.; Plachetka, U., Microelectron. Eng. 2007, 84 (5-8), 945-948.

8. $\quad$ Maruo, S.; Nakamura, O.; Kawata, S., Opt. Lett. 1997, 22 (2), 132-134.

9. Cumpston, B. H.; Ananthavel, S. P.; Barlow, S.; Dyer, D. L.; Ehrlich, J. E.; Erskine, L. L.; Heikal, A. A.; Kuebler, S. M.; Lee, I. Y. S.; McCord-Maughon, D.; Qin, J.; Rockel, H.; Rumi, M.; Wu, X.-L.; Marder, S. R.; Perry, J. W., Nature 1999, 398 (6722), 51-54.

10. Ivanova, E. P.; Hasan, J.; Webb, H. K.; Gervinskas, G.; Juodkazis, S.; Truong, V. K.; Wu, A. H.; Lamb, R. N.; Baulin, V. A.; Watson, G. S.; Watson, J. A.; Mainwaring, D. E.; Crawford, R. J., Nature communications 2013, 4, 2838.

11. Huang, Z. P.; Geyer, N.; Werner, P.; de Boor, J.; Gosele, U., Adv. Mater. 2011, 23 (2), 285-308.

12. Hildreth, O. J.; Rykaczewski, K.; Fedorov, A. G.; Wong, C. P., Nanoscale 2013, 5 (3), 961-970. 
13. Lai, C. Q.; Cheng, H.; Choi, W. K.; Thompson, C. V., The Journal of Physical Chemistry C 2013, 117 (40), 20802-20809.

14. Nguyen, N. T.; Wereley, S. T., Fundamentals and Applications of Microfluidics. Artech House: 2002.

15. Geyer, N.; Fuhrmann, B.; Huang, Z.; de Boor, J.; Leipner, H. S.; Werner, P., The Journal of Physical Chemistry C 2012, 116 (24), 13446-13451.

16. Geyer, N.; Fuhrmann, B.; Leipner, H. S.; Werner, P., ACS Appl. Mater. Interfaces 2013, 5 (10), 4302-4308.

17. Yu, N.; Capasso, F., Nat. Mater. 2014, 13 (2), 139-150.

18. Lin, D.; Fan, P.; Hasman, E.; Brongersma, M. L., Science 2014, 345 (6194), 298-302.

19. Kildishev, A. V.; Boltasseva, A.; Shalaev, V. M., Science 2013, 339 (6125).

20. Sun, S.; Yang, K.-Y.; Wang, C.-M.; Juan, T.-K.; Chen, W. T.; Liao, C. Y.; He, Q.; Xiao, S.; Kung, W.T.; Guo, G.-Y.; Zhou, L.; Tsai, D. P., Nano Lett. 2012, 12 (12), 6223-6229.

21. Davis, B. L.; Hussein, M. I., Phys. Rev. Lett. 2014, 112 (5), 055505.

22. Maldovan, M., Nature 2013, 503 (7475), 209-217.

23. Zhang, S.; Yin, L.; Fang, N., Phys. Rev. Lett. 2009, 102 (19), 194301. 
Figure 1: M.A. Otte et al.

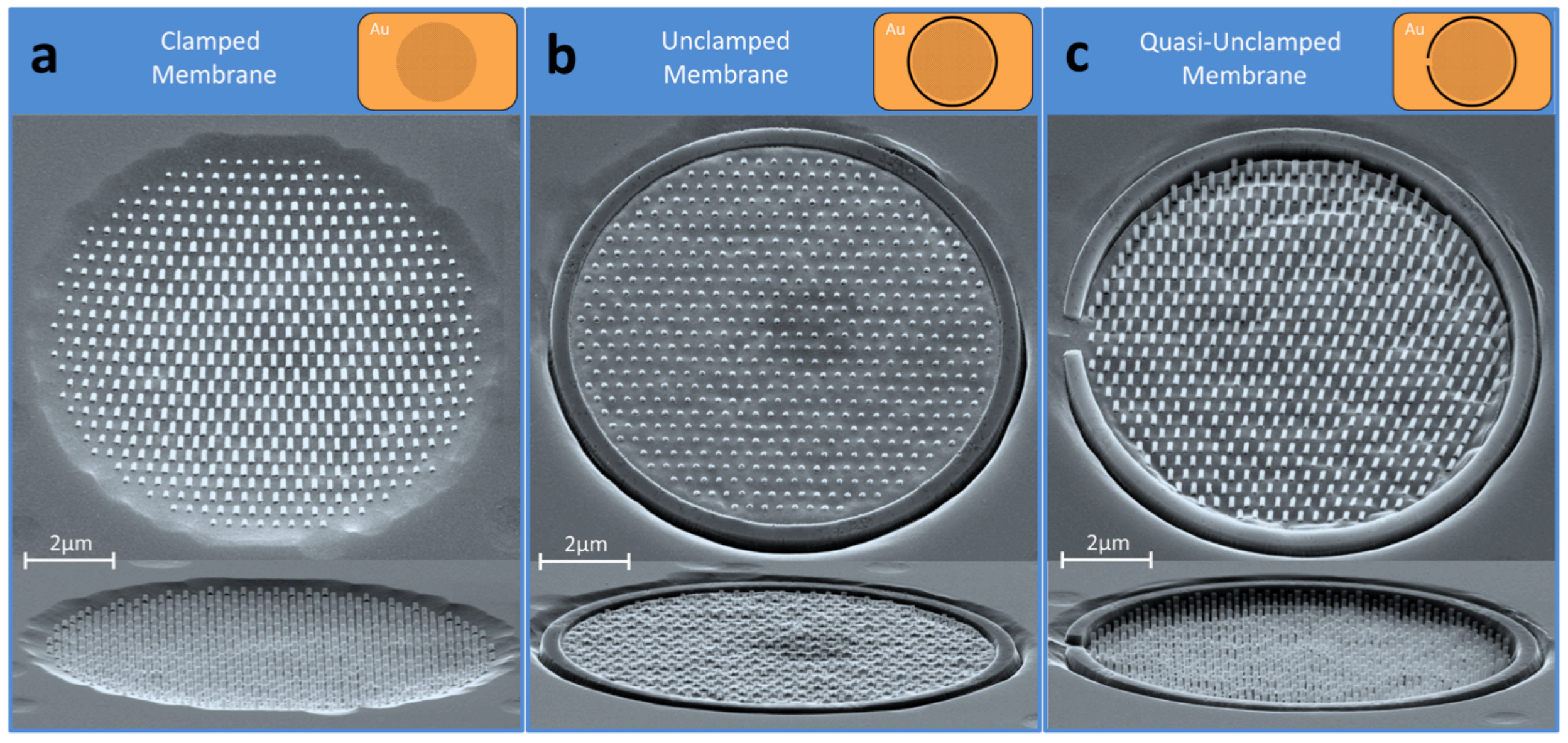


Figure 3: M.A. Otte et al.
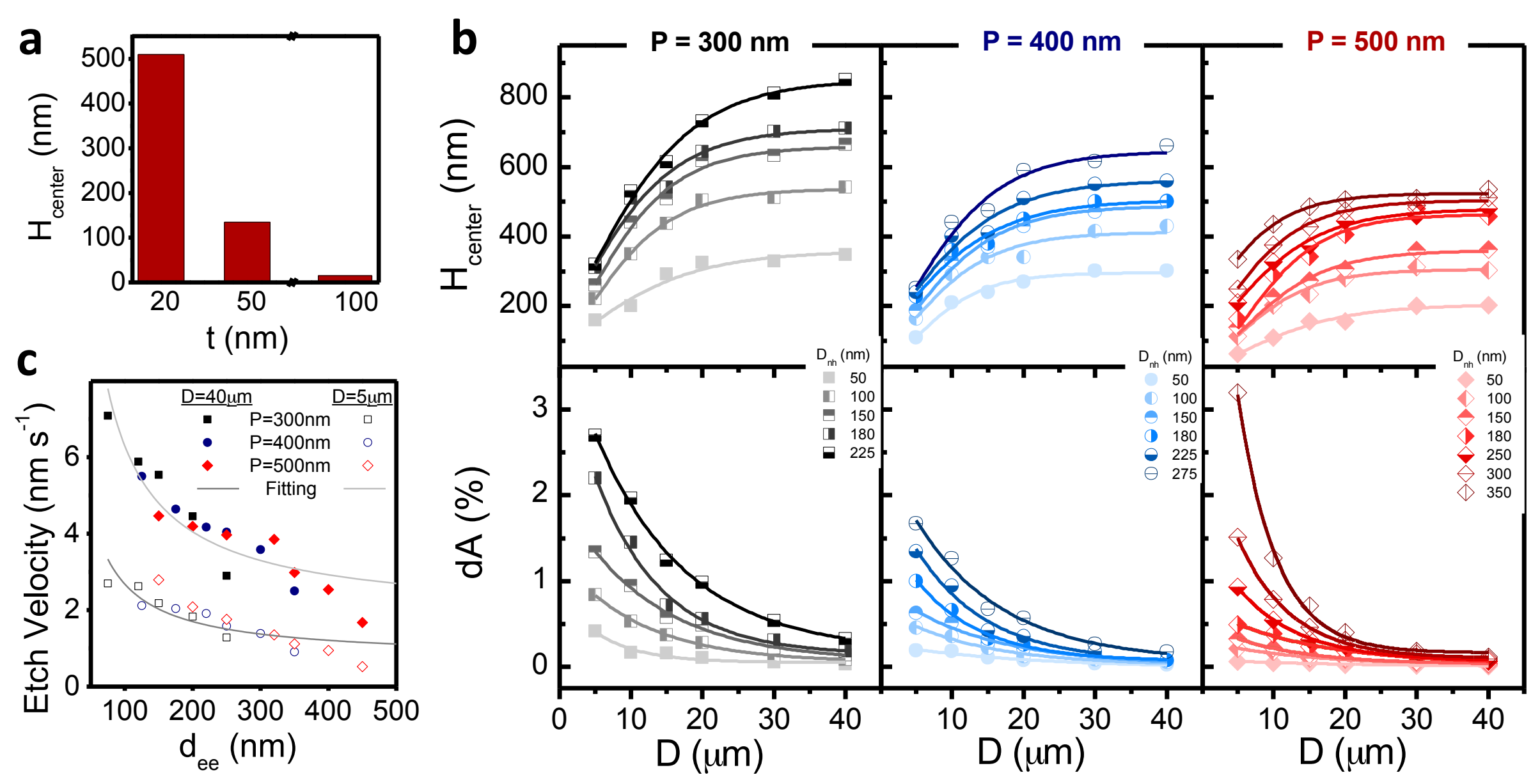
Figure 4: M.A. Otte et al.
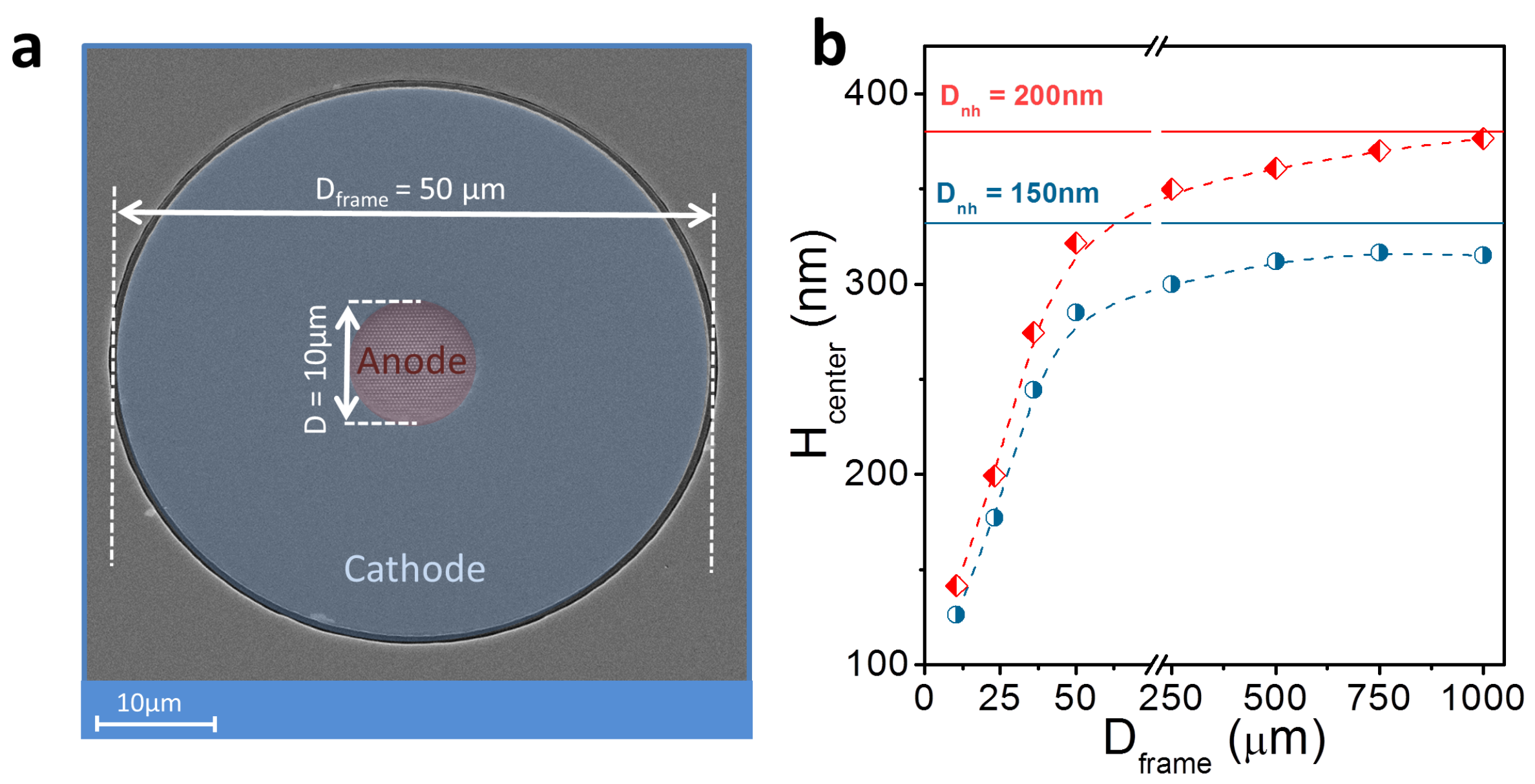


\section{Figure 5: M.A. Otte et al.}
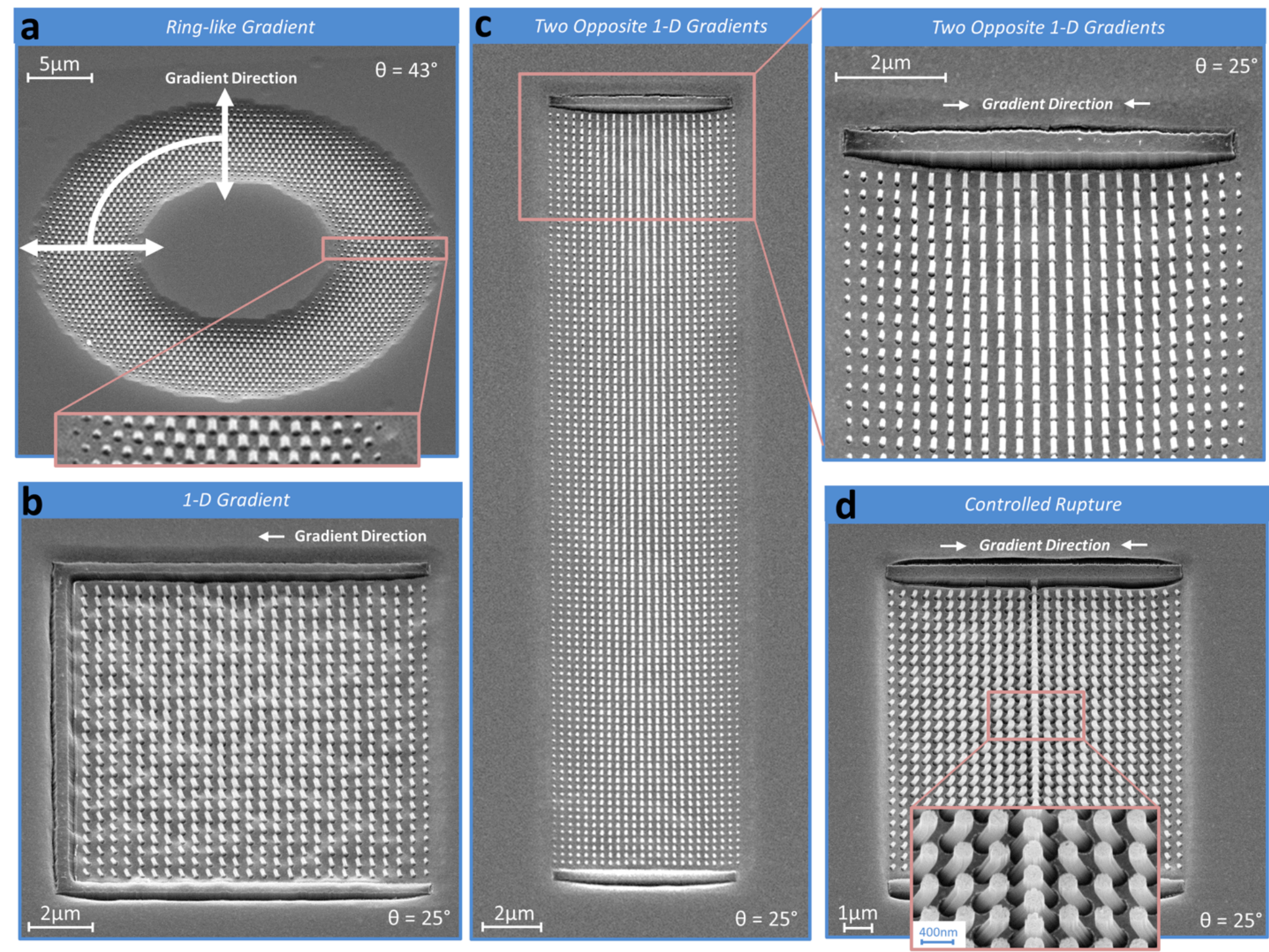
Figure 2: M.A. Otte et al.

a

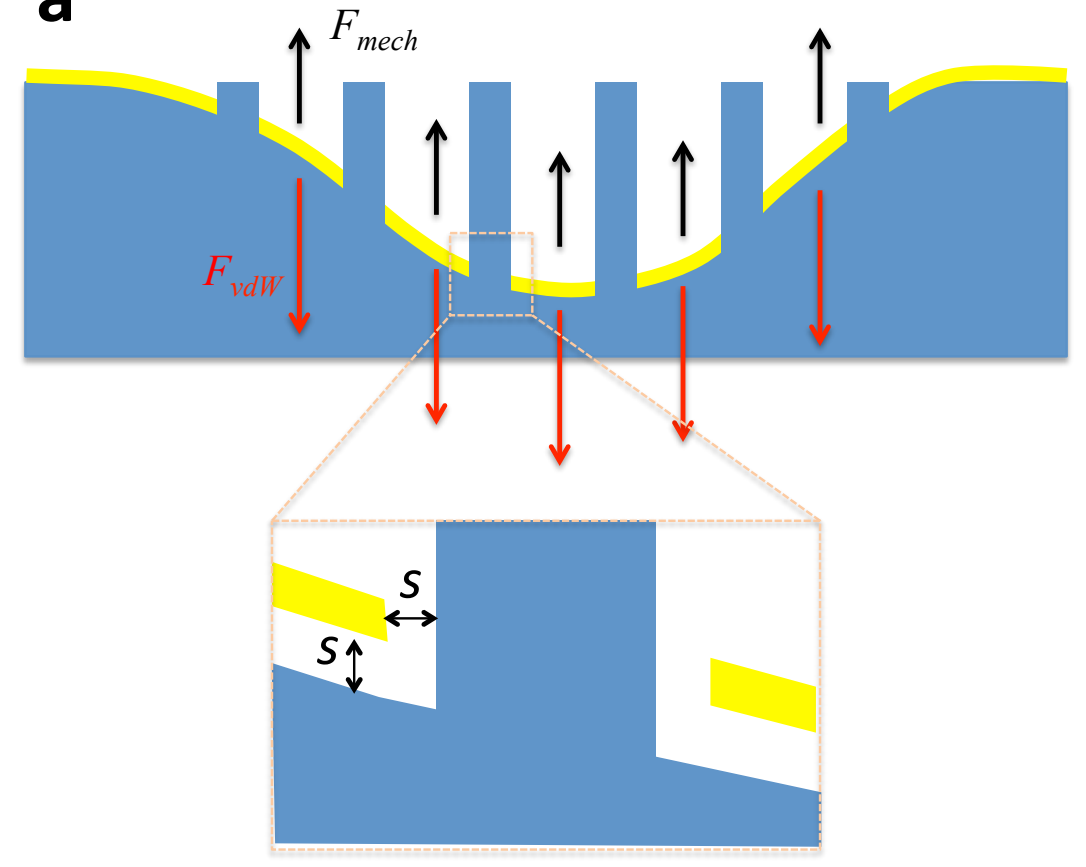

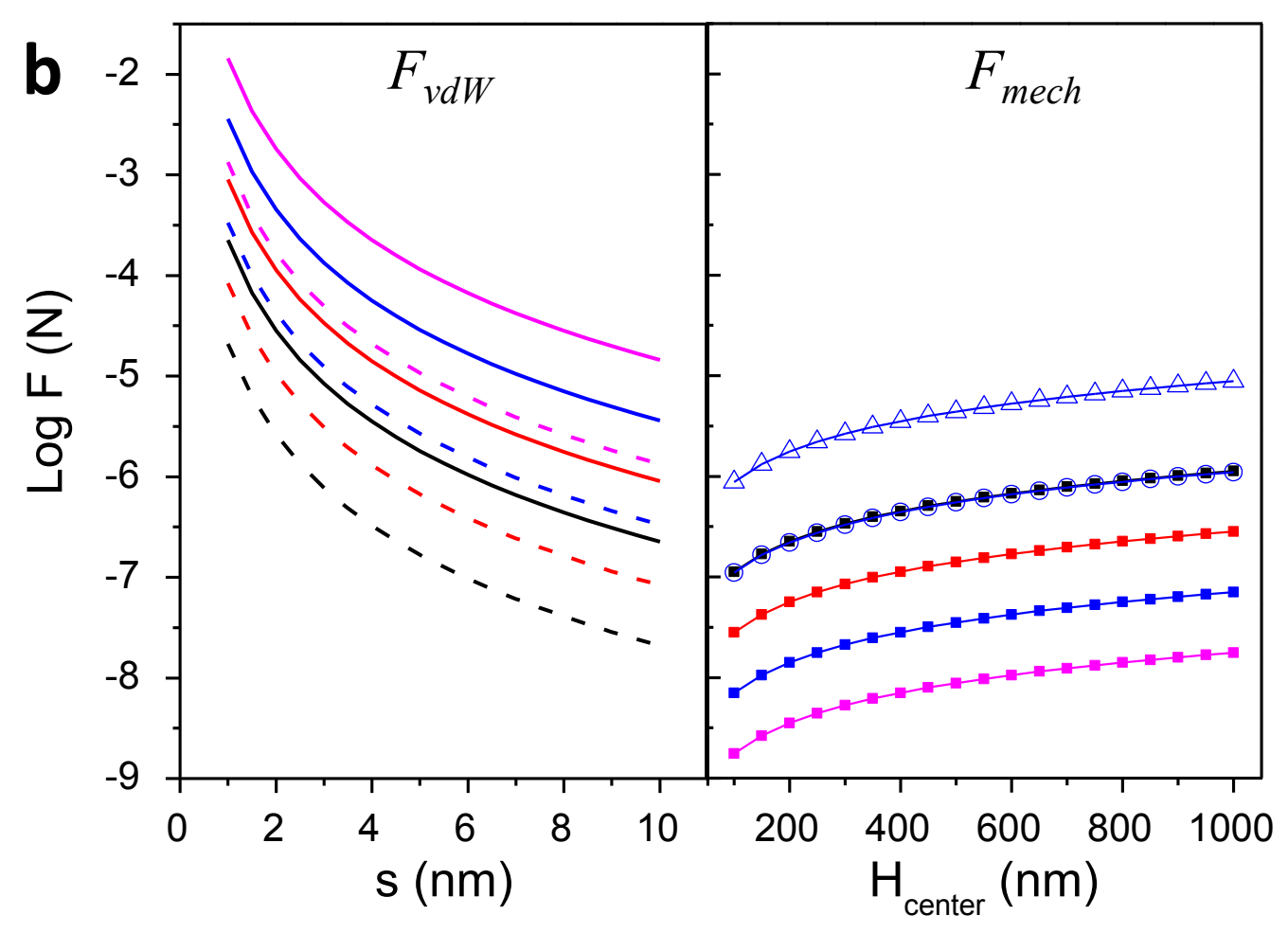


Figure 3: M.A. Otte et al.
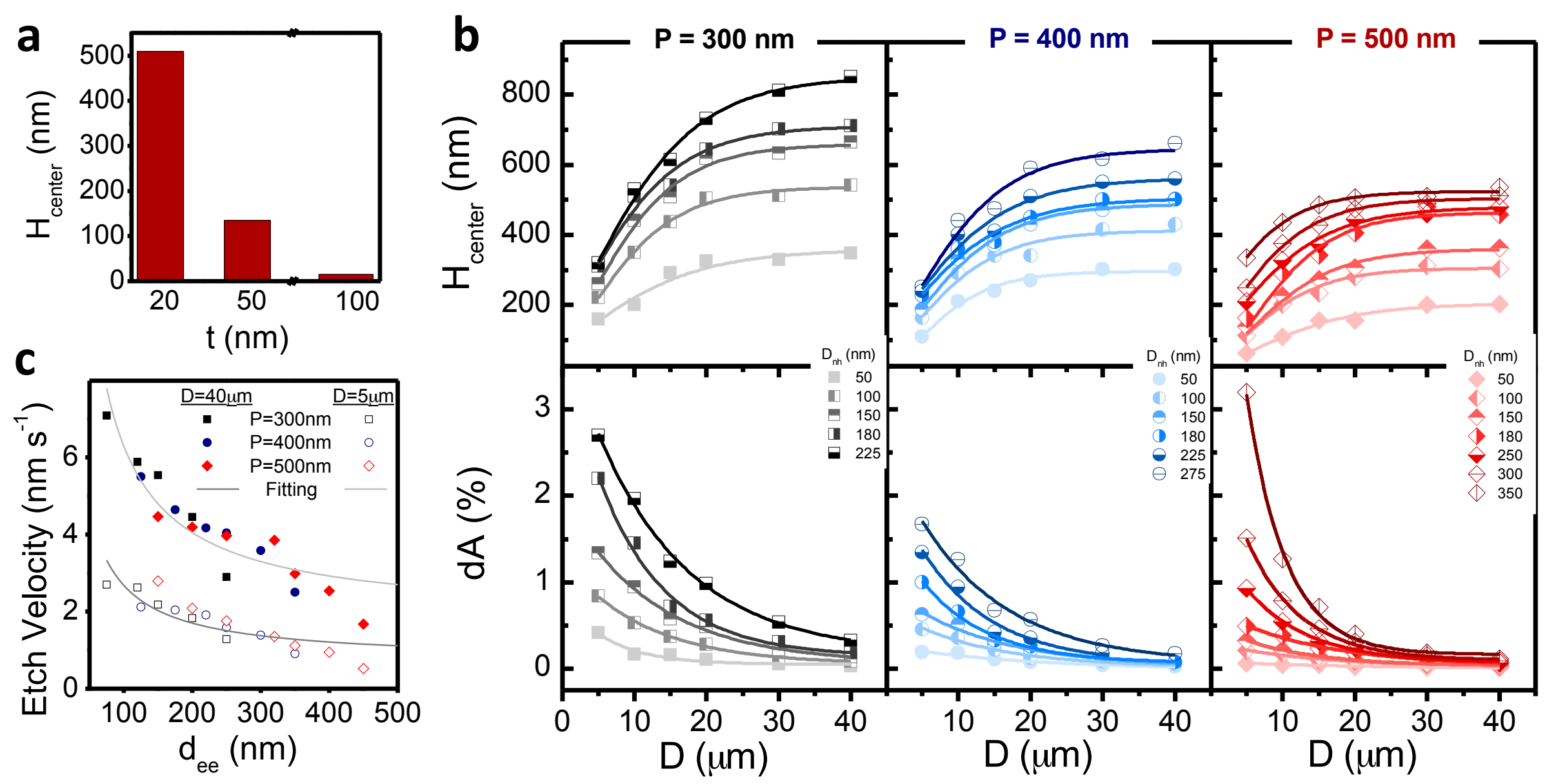
Figure 4: M.A. Otte et al.
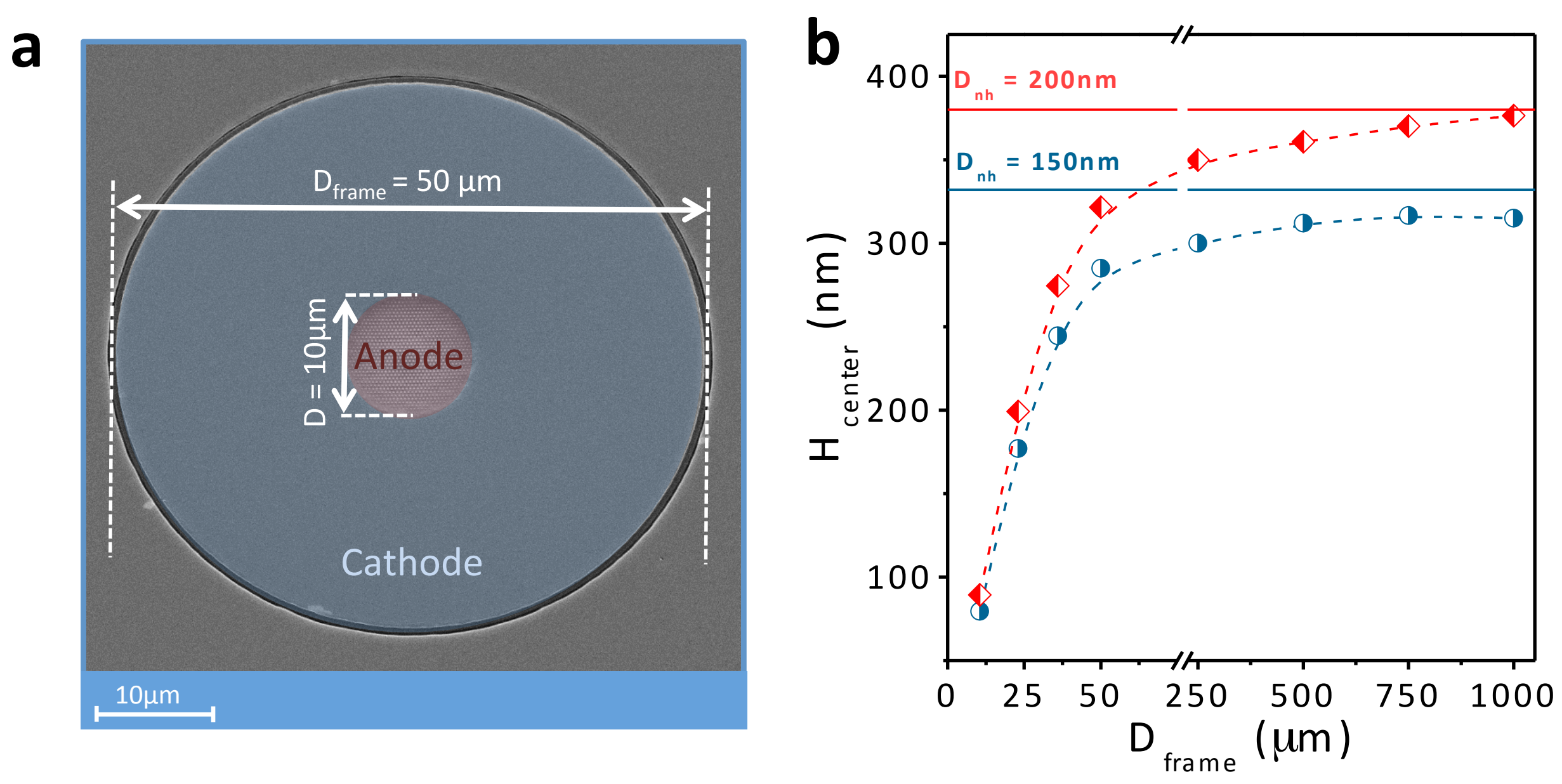
Figure 5: M.A. Otte et al.
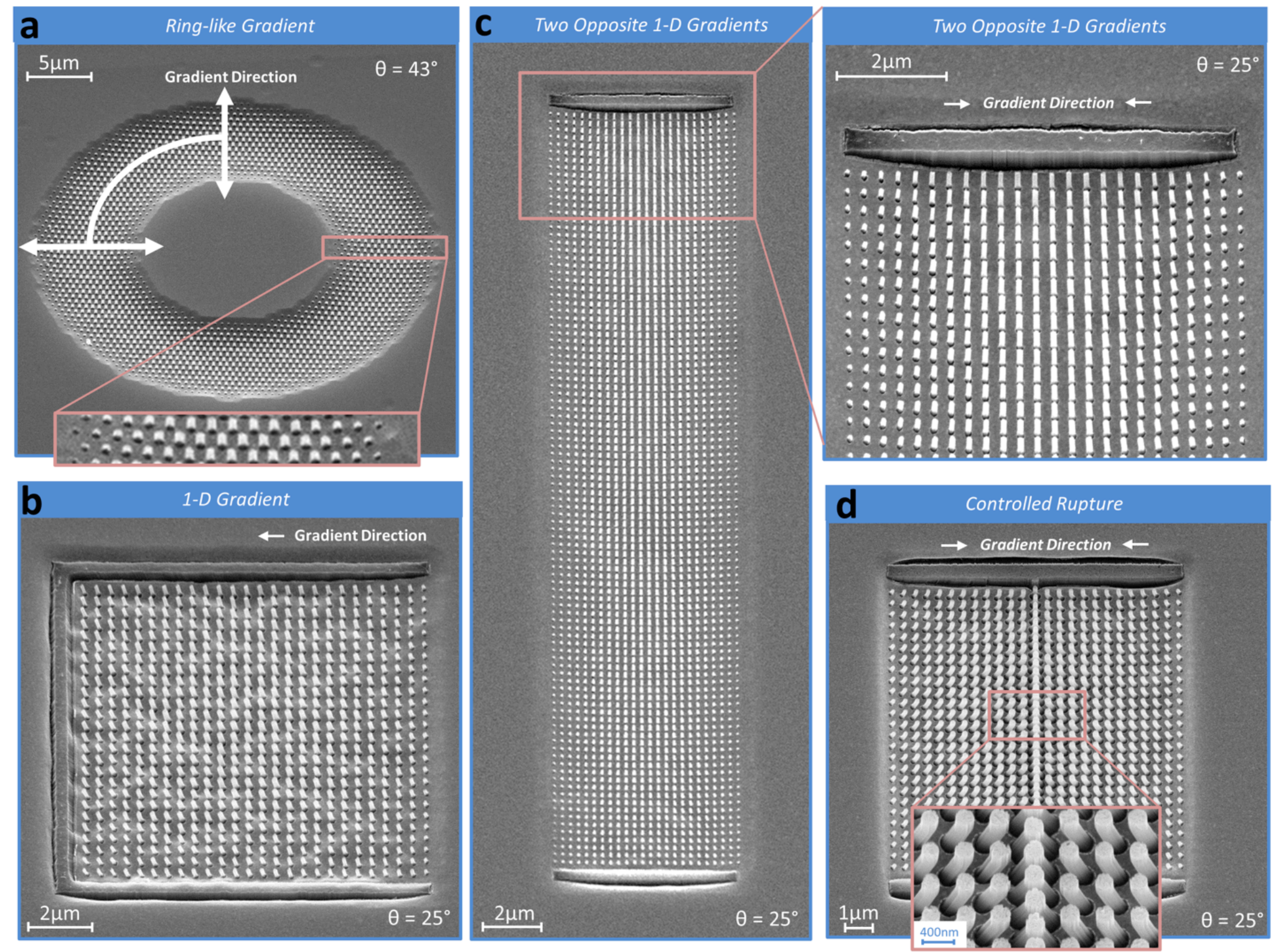